\title{
SIMULATION OF STRESS-STRAIN STATE OF LONG RAILS AFTER
}

\section{DIFFERENTIAL QUENCHING}

\section{VALERY SEMENOVICH KOSSOV ${ }^{1}$, GRIGORY MIKHAYLOVICH VOLOKHOV ${ }^{2}$, MIKHAIL NIKOLAEVICH OVECHNIKOV ${ }^{3}$, ANDREY ALEKSANDROVICH LUNIN ${ }^{4}$ \& ANDREY LEONIDOVICH PROTOPOPOV ${ }^{5}$}

${ }^{1}$ Doctor of Engineering, professor \& General Director of Joint Stock Company "Scientific-Research and Design-Technology Institute of Rolling Stock” (JSC “VNIKTI”), Kolomna, Moscow, Russia

${ }^{2}$ Doctor of Engineering, Head of the Department of Rolling Stock Dynamics and Strength and Infrastructure, (JSC “VNIKTI”), Kolomna, Moscow, Russia

${ }^{3}$ Doctor of Engineering, Head of the Laboratory of Strength Analysis, (JSC “VNIKTI”), Kolomna, Moscow, Russia

${ }^{4}$ Candidate of Engineering, Deputy General Director, (JSC “VNIKTI”), Kolomna, Moscow, Russia

${ }^{5}$ Senior Research Scientist, (JSC “VNIKTI”), Kolomna, Moscow, Russia

\section{ABSTRACT}

Papers of the researchers in the field of rail quenching technology are analyzed. Mathematical models of phase transformations and metal quenching of rails are considered. The directions of further studies of the mechanical properties of rails are indicated for the purpose of the evaluation of their service life in order to ensure long-term and safe operation.

KEYWORDS: Rail Properties; Models of Phase Transformation and Quenching; Joint Task; Strain \& Residual Stress

Received: Mar 22, 2019; Accepted: Apr 12, 2019; Published: Jun 12, 2019; Paper Id.: IJMPERDJUN2019170

\section{INTRODUCTION}

The safety of railway transport operation depends largely on the reliability and durability of railway rails, as the main supporting element of the superstructure. From the point of view of their fatigue strength, this property is significantly affected by the presence of irregularities along the length and the distribution of residual stress fields over a rail section, which occur as a result of the manufacturing process.

The aim of this work is to study the stress-strain state of long rails by a finite element simulation of the process of its thermal differential quenching. The mathematical model takes into account: complex physical phenomena occurring in the rail metal, structural changes, significant non-linearity of physical and mechanical properties under conditions of high temperatures. As a result of the carried out studies, distributions of the residual stresses and strains in the rail under different conditions of its differential quenching are obtained.

\section{STUDY OBJECT AND ITS PROPERTIES}

The object of this study is a rail, type Р65, category ДТ350, made of steel grade Э76Ф, with the length of $100 \mathrm{~m}$ and the cross-sectional dimensions and properties according to GOST R 51685 (2014). The physical and mechanical properties and the parameters of the continuous cooling transformation (CCT) diagram of phase transformations of the indicated above steel during the decomposition of the overcooled austenite are presented 
in(Polevoy, 2018).

\section{METHOD OF PROBLEM SOLUTION}

The numerical method for the simulation of the rail quenching process implies the problem solution of transient heat conduction, when the temperature in the body under study changes over time as a result of the convective exchange of its surface with an external moving heat transfer medium. The scheme of air blow-off of a long rail surface adopted in this paper is shown in Figure 1. A similar scheme is given in (Polevoy, 2018). Air is used as a heat transfer medium during the differential quenching.

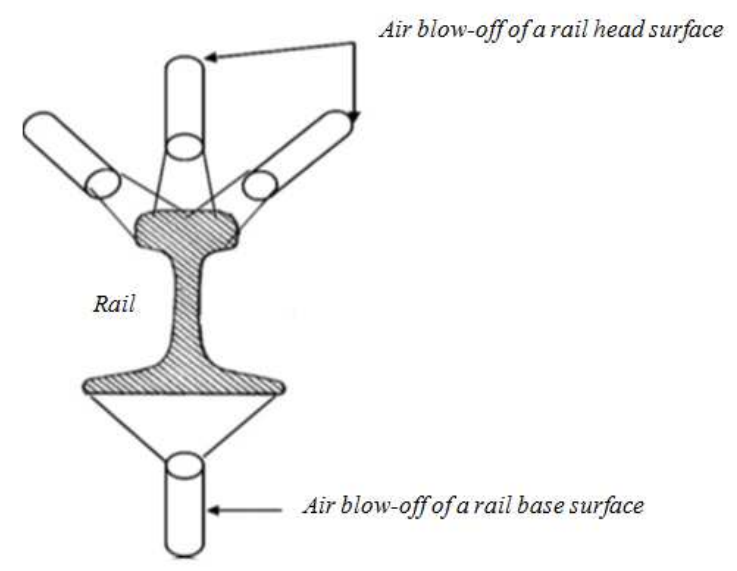

Figure 1: The Scheme of the Cooling of the Rail Surfaces During its Differential Quenching

As shown in figure 1 the essence of the method of the differential quenching consists in the treatment with air flow of different speed (regulated by the pressure in the air supply network) of the surfaces of the rail head and its base. In the mathematical model of this study, this was taken into account by varying the heat transfer coefficient over the corresponding surfaces.

The quenching is characterized by an uneven temperature variation in the volume of hot metal over time and, as a result, by structural transformations (possible presence of different phases of metal state - austenite, perlite, bainite and martensite). Moreover, due to the specified uneven temperature and the phase state of the material, some irreversible plastic strains can occur, which lead to the final residual stresses and distortions of the rail straightness after the quenching. As a method for this problem solution, the algorithm of the joint task was used, that is, at each time step of the transient heat conduction calculation, the problem of the stress-strain state calculation was solved taking into account different phase states of the rail metal.

\section{MATHEMATICAL MODEL}

Cooling conditions during the rail quenching allow the use of the principle of symmetry in the finite element model forming. In figure 2 the finite element model of a half of a hundred-meter rail is shown. It shows the imposed kinematic boundary conditions. In the plane of symmetry, some movements of the rail in the longitudinal direction are fixed. On the rail base, some movements in the vertical direction during the quenching are fixed. With further rail cooling after quenching the restrictions on the rail base are removed. In the section plane and along the rail length, on the basis of the series of calculations, the necessary size of the finite element was selected to ensure the accuracy of the obtained 
calculation results.

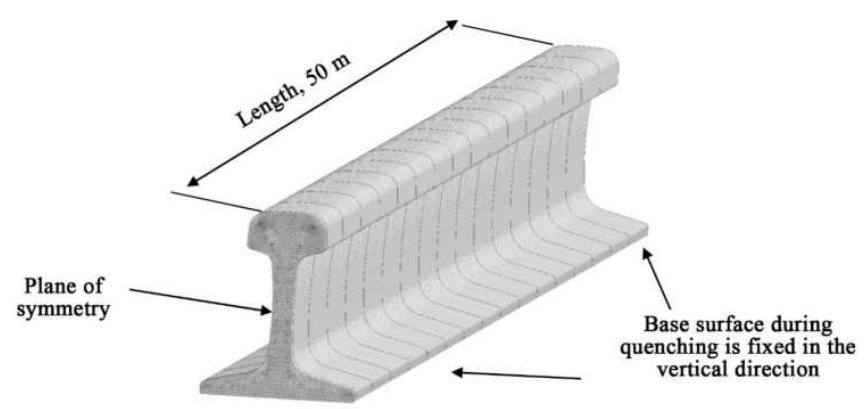

Figure 2: The Finite Element Model of a Rail Half with the Imposed Boundary Conditions

\section{Heat Exchange and Quenching Mode Conditions}

The heat exchange conditions were chosen on the basis of the research results given in (Polevoy, 2018) according to the condition that at the initial heat treatment temperatures of $850^{\circ} \mathrm{C}$ and the corresponding speeds in the range of $2 \ldots 3,3$ degrees/s, the cooling leads to the most sustainable results, concerning the uniform pearlite microstructure of the entire rail section and the required mechanical properties of the rail head running surface region.

In solving the problem of the transient heat conduction, a boundary condition was used under which the dependence of the heat transfer between the surface of a body and the environment was set by the Newton-Richmann law:

$$
q=\alpha\left(T_{s}-T_{a}\right)
$$

Where $q$ - heat flow density, W/ $\mathrm{m}^{2}$;

- $\quad \boldsymbol{\alpha}$ - heat transfer coefficient $\mathrm{W} /\left(\mathrm{m}^{2} \cdot{ }^{\circ} \mathrm{C}\right)$;

- $\quad \boldsymbol{T}_{\boldsymbol{s}}$ - body surface temperature, ${ }^{\circ} \mathrm{C}$;

- $\boldsymbol{T}_{\boldsymbol{a}}$ - ambient temperature, ${ }^{\circ} \mathrm{C}$.

In the calculations the initial temperature of the rail and, accordingly, of its surface was assumed to be equal to $850^{\circ} \mathrm{C}$, and the ambient temperature - to $20^{\circ} \mathrm{C}$. The quenching time was taken to be equal to $120 \mathrm{~s}$ (Polevoy, 2018). During the quenching in the series of calculations, the heat transfer coefficient was chosen so as to ensure the cooling rates over the rail head indicated above. In the calculations over the base surface the heat transfer coefficient varied in percentage terms to the heat transfer coefficient value on the rail head surface, what was necessary to study the effect of the structural changes synchronization in the material on the final stress-strain state of the rail after its quenching.

\section{CALCULATION RESULTS}

Figure 3 presents the results of the final distortion of the rail after its cooling to the ambient temperature in the form of the dependence of vertical displacements of its ends relative to the middle of the rail on the percentage ratio of the heat transfer coefficients when quenched at the rail base and at the rail head:

$$
K=\frac{\alpha_{b}}{\alpha_{h}} \cdot 100 \%
$$


where $\alpha_{b}$ - heat transfer coefficient value whenquenched at the rail base;

$\alpha_{h}$ - heat transfer coefficient value when quenched at the rail head.

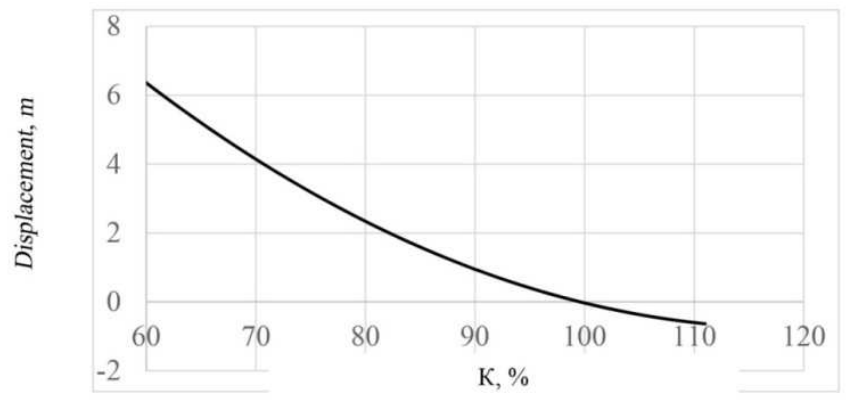

Figure 3: The Dependence of the Rail Ends Distortion Relative to its Midpoint on the Percentage Ratio of the Heat Transfer Coefficients

It can be seen from the figure that there is the relation between the heat transfer coefficients on the rail head and the rail base, at which the rail takes the straight form after the quenching.

Figure 4 shows the distribution of the residual longitudinal stresses perpendicular to the rail cross section after the quenching.

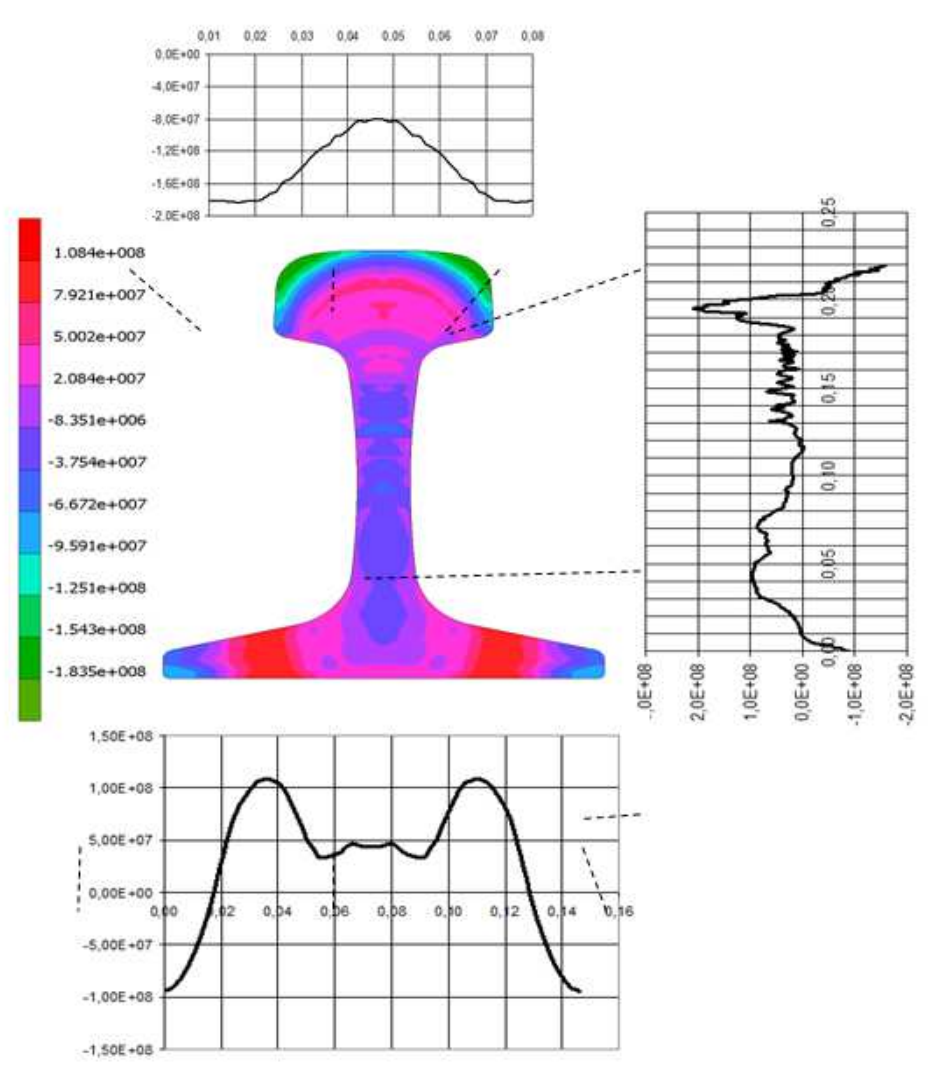

Figure 4: The Field of the Longitudinal Residual Stresses $\sigma(\mathrm{Pa})$ in the Middle Section of the 100-Meter Rail After its Differential Quenching

The analysis of the figure shows that the level of the residual stresses along the rail section varies from -185 to $108 \mathrm{MPa}$. On the head surface, they are compressive and they vary in the range from -80 to -185 MPa. On the base surface there are tensile stresses of $100 \mathrm{MPa}$ level in its midpoint and compressive stresses on the base edges (about -100 MPa). 
The stresses are almost zero in the middle of the rail web.

\section{DISCUSSIONS OF THE RESULTS AND THE DIRECTIONS OF FURTHER STUDIES}

As the results of the numerical studies show, after the implementation of the differential quenching of long rails without cold straightening, in general, a favorable pattern of the residual stress field distribution is formed: the compressive stresses were observed near the surface of the rail head and along the edges of its base. The stresses are almost zero in the middle of the rail web. However, after the process of the cold straightening on a mangle, the pattern of the stress distribution with the final significant distortion of the rail in its vertical plane after the quenching may undergo significant changes. Near the running surface, where there were compressive stresses, some tensile stresses may occur (Zolotarskiy, 1976). This points out the necessity of further optimization of the quenching process to minimize the distortion of the rail.

With the satisfactory result achieved for the global residual strain of the rail from the impact of the quenching mode, there is a necessity for more detailed studies of the distribution of the residual stresses and the hardness over its cross section (Borts, 2009). On this basis, further numerical studies of the quenching mode optimization and the subsequent straightening of rails in order to meet the acceptance requirements (GOST R 51685-2013, 2014) are expected.

\section{CONCLUSIONS}

As the results of the numerical studies show, after the implementation of the differential quenching of long rails without cold straightening, in general, a favorable pattern of the residual stress field distribution is formed: the compressive stresses were observed near the surface of the rail head and along the edges of its base. The stresses are almost zero in the middle of the rail web. However, after the process of the cold straightening on a mangle, the pattern of the stress distribution with the final significant distortion of the rail in its vertical plane after the quenching may undergo significant changes. Near the running surface, where there were compressive stresses, some tensile stresses may occur (Zolotarskiy, 1976). This points out the necessity of further optimization of the quenching process to minimize the distortion of the rail.

With the satisfactory result achieved for the global residual strain of the rail from the impact of the quenching mode, there is a necessity for more detailed studies of the distribution of the residual stresses and the hardness over its cross section (Borts, 2009). On this basis, further numerical studies of the quenching mode optimization and the subsequent straightening of rails in order to meet the acceptance requirements (GOST R 51685-2013, 2014) are expected.

\section{REFERENCES}

1. GOST R 51685-2013. (2014).Railway rails. General specifications (EN 13674-1: 2011, NEQ). Moscow: Standardinform, 101.

2. Polevoy, E.V. (2018). Development of the resource-saving technology for the differential heat treatment of long railway rails: dis. ... cand. tech. sciences. Novokuznetsk, 132.

3. Zolotarskiy,A.F. (1976).Heat-treated rails. Moscow: Transport, 264.

4. Ghosh, S. Inertia Effect Under Couple Stress Fluid In Laminar Flow Condition In Journal Bearing.

5. Borts, A.I. Shur, E.A., Reyhart, V.A., Bazanov,Yu.A. (2009). The test results of the rails subjected to the differential quenching from rolling heat, the influence of the production technology features on their properties. Industrial transport XXI century, 4 , $32-36$. 
